\title{
Multicenter phase II study of biweekly CAPIRI plus bevacizumab as second-line therapy in patients with metastatic colorectal cancer (JSWOG-C3 study)
}

\author{
Nobuaki Suzuki ${ }^{1}$ (1) S Shoichi Hazama ${ }^{1,2} \cdot$ Takeshi Nagasaka $^{3} \cdot$ Hiroaki Tanioka $^{3}$ - Yasuo Iwamoto ${ }^{4}$ - Yuji Negoro ${ }^{5}$. \\ Masami Yamauchi ${ }^{6} \cdot$ Michiya Kobayashi $^{7} \cdot$ Hiroshi Okuda ${ }^{8} \cdot$ Noriaki Fujishima $^{9} \cdot$ Taku Nishimura $^{10}$. \\ Naoki Yamanaka ${ }^{11} \cdot$ Kazuhiro Toyota $^{12} \cdot$ Yoshiko Mori $^{13} \cdot$ Yuki Nakagami $^{1,2} \cdot$ Mototsugu Shimokawa $^{14}$. \\ Hiroaki Nagano ${ }^{1} \cdot$ Masazumi Okajima $^{15}$
}

Received: 22 December 2018 / Accepted: 18 May 2019 / Published online: 29 May 2019

(C) The Author(s) 2019

\begin{abstract}
Background Triweekly capecitabine plus irinotecan (CAPIRI) was not a replacement for fluorouracil, leucovorin, and irinotecan (FOLFIRI) in the treatment of metastatic colorectal cancer (mCRC) because of the potential for greater toxicity. Recently, it has reported that mCAPIRI is well tolerated and non-inferior to FOLFIRI. In this study, we conducted a multicenter phase II trial to assess the efficacy and safety of biweekly CAPIRI plus bevacizumab as second-line chemotherapy for mCRC with reduced toxicity and preserved efficacy.

Methods Patients with mCRC who had received prior chemotherapy, including oxaliplatin-based regimens, were eligible for this study. The treatment protocol administered capecitabine at $1000 \mathrm{mg} / \mathrm{m}^{2}$ twice daily from the evening of day 1 to the morning of day 8 , intravenous irinotecan at $150 \mathrm{mg} / \mathrm{m}^{2}$ on day 1 , and bevacizumab at $10 \mathrm{mg} / \mathrm{kg}$ on day 1 every 2 weeks. Primary endpoints for this study were progression-free survival (PFS) and safety. Secondary endpoints were overall survival (OS), time to treatment failure, response rate (RR), and disease control rate (DCR).

Results Fifty-one patients were enrolled in this study. Median PFS was 5.5 months [95\% confidence interval (CI) $4.23-$ 7.40 months], and median OS was 13.5 months (95\% CI 11.57-20.23 months). The RR was $14.6 \%$ (95\% CI 6.5-28.4\%), and the DCR was 66.7\% (95\% CI 51.5-79.2\%). Hypertension was the most common Grade 3 adverse event (27.5\%), followed by neutropenia (17.6\%). Only two patients suffered from grade 3 hand-foot syndrome.

Conclusions In mCRC patients, biweekly CAPIRI + bevacizumab appears effective and feasible as a second-line chemotherapy with relatively low toxicities, and has potential as a useful substitute for FOLFIRI + bevacizumab.
\end{abstract}

Keywords Bevacizumab $\cdot$ CAPIRI $\cdot$ Biweekly $\cdot$ Second line $\cdot$ mCRC

\section{Introduction}

Colorectal cancer (CRC) is one of the leading causes of cancer-related deaths and the most common cancer type, with more than one million new cases diagnosed annually worldwide [1-3]. In recent years, new regimens for colon cancer combining chemotherapy and biological agents

Nobuaki Suzuki and Shoichi Hazama contributed equally to this study.

Nobuaki Suzuki

nobusuzu@yamaguchi-u.ac.jp

Extended author information available on the last page of the article have improved the overall survival (OS) and progressionfree survival (PFS) [4-7]. Standard treatments for patients with metastatic CRC (mCRC) usually consist of combination chemotherapy based on fluorouracil or capecitabine plus either oxaliplatin or irinotecan, and a targeted agent such as bevacizumab, cetuximab, or panitumumab [8-10]. The most commonly used chemotherapy regimens are fluorouracil with folinic acid plus oxaliplatin (FOLFOX), fluorouracil with folinic acid plus irinotecan (FOLFIRI), and capecitabine plus oxaliplatin (XELOX). Particularly as a second-line regimen, triweekly capecitabine plus irinotecan (CAPIRI) was not a replacement for fluorouracil, leucovorin, and irinotecan (FOLFIRI) in the treatment of metastatic colorectal cancer (mCRC) because of the potential for 
greater toxicity [11-13]. However, some phase II trials have suggested that a modified reduced-dose CAPIRI regimen (irinotecan $200 \mathrm{mg} / \mathrm{m}^{2}$ on day 1 plus capecitabine $800 \mathrm{mg}$ / $\mathrm{m}^{2}$ twice daily on days $1-14$ ) once every 3 weeks offered favorable tolerability and efficacy in the second-line setting [14]. Recently, an Asian phase III trial, the AXEPT study has reported Mcapiri, is well tolerated and non-inferior to FOLFIRI [15]. We also found another phase I/II study of biweekly capecitabine and irinotecan plus bevacizumab as second-line chemotherapy in patients with mCRC, but that was conducted at only one high-volume center hospital [16]. However, we could not find any multicenter trials for biweekly CAPIRI plus bevacizumab $(10 \mathrm{mg} / \mathrm{kg})$ in a secondline setting. From the perspective of patient care and scheduled administration of chemotherapy, some advantages are seen in a biweekly regimen, which is easier to manage than a weekly or triweekly regimen, but the usefulness of this method is still unclear. Therefore, given the current situation in Japan in which oxaliplatin-based chemotherapy is provided as the primary therapy, we have envisioned a multicenter phase II study to assess the efficacy and safety of biweekly CAPIRI plus bevacizumab $(10 \mathrm{mg} / \mathrm{kg})$ as secondline chemotherapy for mCRC. In relation to a biweekly regimen for capecitabine, we have experience with achieving acceptable toxicity and good efficacy in a trial for another CAPEOX regimen using a biweekly capecitabine regimen (capecitabine at $1000 \mathrm{mg} / \mathrm{m}^{2}$ twice a day on days 1-7) [17]. Since the key drug in the second-line therapy is considered to be irinotecan, a CAPIRI regimen that can be administered every other week using irinotecan at $150 \mathrm{mg} / \mathrm{m}^{2}$ as the standard dose in Japan with an oral fluoropyrimidine is important. In this study, we, therefore, conducted a multicenter phase II trial to assess the efficacy and safety of biweekly CAPIRI plus bevacizumab as second-line chemotherapy for mCRC with reduced toxicity and preserved efficacy.

\section{Patients and methods}

\section{Study design}

This single-arm, phase II, multi-institutional clinical trial was conducted to determine the efficacy and safety of a combination regimen of bevacizumab $(10 \mathrm{mg} / \mathrm{kg})$ with biweekly CAPIRI as second-line chemotherapy in patients with mCRC. The study was performed in accordance with the Declaration of Helsinki and the ethical guidelines for clinical studies. The study protocol was approved by the institutional review board of Yamaguchi University (H23-182), and was then started after approval from the relevant institutional review boards at each of the participating institutions. Trial registration: this study has been registered in the University
Hospital Medical Information Network (UMIN) Clinical Trials Registry as UMIN 000009280.

\section{Inclusion criteria}

Eligibility criteria for patients were as follows: histologically proven colorectal 5 adenocarcinoma; unresectable or recurrent disease; a measurable lesion confirmed 28 days prior to enrollment; prior chemotherapy for metastatic or recurrent disease, including oxaliplatin-based regimens with bevacizumab; preserved organ functions [neutrophil count $\geq 1.5 \times 10^{3} / \mathrm{mm}^{3}$; platelets $\geq 10.0 \times 10^{3} / \mathrm{mm}^{3}$; hemoglobin $\geq 9.0 \mathrm{~g} / \mathrm{dl}$; total bilirubin $\leq 1.5 \mathrm{mg} / \mathrm{dl} ;$ AST/ALT $\leq 100 \mathrm{IU} / \mathrm{l}$; creatinine $\leq 1.5 \mathrm{mg} / \mathrm{dl}$; urinary protein $\leq 1+$ ]; Eastern Cooperative Oncology Group performance status (ECOG PS) $0-1$; expected survival $\geq 3$ months; age $\geq 20$ years; no double cancer; and provision of written, informed consent to participate. On the other hand, the dose adjustment based on the measurement result of UGT1A1 gene polymorphism before the first dose administration is not indispensable.

\section{Exclusion criteria}

Exclusion criteria for patients were as follows: severe peritoneal ascites or pleural effusion; jaundice; intestinal obstruction; severe renal failure; brain tumor and brain metastasis recognized on imaging; duplicated cancers with a diseasefree period of less than 5 years; radiation therapy carried out within 4 weeks before registration; antithrombotic agent administered to thrombosis within 10 days before registration; unhealed traumatic fracture; uncontrollable hypertension; history of myocardial infarction within 1 year before registration; and patients judged otherwise inappropriate by a doctor.

\section{Treatment plan}

Treatment consisted of irinotecan at $150 \mathrm{mg} / \mathrm{m}^{2}$ as an intravenous infusion on day 1 every 2 weeks, capecitabine at $1000 \mathrm{mg} / \mathrm{m}^{2}$ twice daily on days $2-8$, followed by 1 week of rest, and bevacizumab at $10 \mathrm{mg} / \mathrm{kg}$ as an intravenous infusion on day 1 every 2 weeks.

Treatment was continued until one of the following occurred: progressive disease, treatment was not resumed even after 28 days from the last administration, administration difficulty due to severe adverse effects, or decision to stop treatment at the discretion of the treating physician. If chemotherapy was delayed, the administration of bevacizumab was also delayed. If irinotecan or bevacizumab was discontinued, capecitabine and irinotecan or capecitabine and bevacizumab were to be continued unless unacceptable toxicity was observed. If capecitabine was interrupted beyond 28 days, treatment could not be continued. 


\section{Assessment}

Tumors were evaluated using computed tomography before initiation of treatment (within 2 weeks), at 4 weeks after initial treatment, and at 6-week intervals (allowance \pm 2 weeks) thereafter. The primary end points of the present study were PFS and safety. The result of complete response or partial response was confirmed after a subsequent minimum of 4 weeks. Secondary end points included OS, time to treatment failure (TTF), response rate (RR), and disease control rate (DCR). RR and DCR assessed by investigators according to RECIST version 1.1 criteria. No independent radiological review committee was established. Adverse events were monitored and graded according to the National Cancer Institute Common Terminology Criteria for Adverse Events version 4.0.

\section{Statistical analyses}

This single-arm phase II study was designed to assess the PFS and safety of a combination regimen of bevacizumab with biweekly CAPIRI as second-line chemotherapy in patients with mCRC. In the previous report, the median PFS with CAPIRI as second-line chemotherapy was 5.1 months [18]. The target sample size of 50 (43 eligible patients and $10 \%$ ineligible patients) was based on expected and threshold PFS of 8.0 and 5.1 months, respectively, with $\alpha=0.05$ and $\beta=0.1$.

Secondary endpoints were OS, TTF, RR, and DCR. Efficacy analyses were performed on the intention-to-treat population, i.e., patients who received at least one course of study medication. Safety analyses were performed on patients who received study medication at least once. Distributions of PFS, OS, and TTF times were estimated using the Kaplan-Meier method. Qualitative variables were described using absolute and relative frequencies. Quantitative variables were described with means, medians, and standard deviation (SD). All analyses were performed using R language (version 3.5.1).

\section{Results}

\section{Baseline characteristics}

A total of 52 patients were enrolled in the study at 13 Japanese centers between January 2013 and July 2015. One patient failed the screening process and did not meet the entry criteria, and so could not be treated within the study regimen. The remaining 51 patients received treatment and were included in the intention-to-treat and safety populations. Patient characteristics are summarized in Table 1. Median patient age was 66 years (range 41-82 years),
Table 1 Patient characteristics $(N=51)$

\begin{tabular}{|c|c|c|}
\hline & $N$ & $\%$ \\
\hline Median age (range), years & $66(41-82)$ & \\
\hline \multicolumn{3}{|l|}{ Sex } \\
\hline Male & 29 & 56.9 \\
\hline Female & 22 & 43.1 \\
\hline \multicolumn{3}{|l|}{ ECOG PS } \\
\hline $\mathrm{PS}=0$ & 42 & 82.4 \\
\hline $\mathrm{PS}=1$ & 9 & 17.6 \\
\hline \multicolumn{3}{|l|}{ Site of primary tumor } \\
\hline Colon & 31 & 60.8 \\
\hline Rectum & 18 & 35.3 \\
\hline Cecum & 2 & 3.9 \\
\hline \multicolumn{3}{|l|}{ Histology of primary tumor } \\
\hline Well differentiated adenocarcinoma & 11 & 21.6 \\
\hline Moderately differentiated adenocarcinoma & 33 & 64.7 \\
\hline Poorly differentiated adenocarcinoma & 3 & 5.9 \\
\hline Others & 4 & 7.8 \\
\hline \multicolumn{3}{|l|}{ Metastatic sites } \\
\hline Liver limited & 13 & 25.5 \\
\hline Other single region & 9 & 17.6 \\
\hline Multiple site & 29 & 56.9 \\
\hline \multicolumn{3}{|l|}{ UGT1A1 status } \\
\hline Wild & 11 & 21.6 \\
\hline *6 Polymorphism & 7 & 13.7 \\
\hline *28 Polymorphism & 4 & 7.8 \\
\hline Compound heterozygote & 0 & 0 \\
\hline Unmeasured & 15 & 29.4 \\
\hline \multicolumn{3}{|l|}{ Previous therapy } \\
\hline $\begin{array}{l}\text { Previous therapy with panitumumab or cetuxi- } \\
\text { mab }\end{array}$ & 16 & 31.4 \\
\hline Previous therapy with bevacizumab & 31 & 60.8 \\
\hline
\end{tabular}

ECOG Eastern Cooperative Oncology Group, PS performance status

and patients comprised 29 males (56.9\%) and 22 females (43.1\%). ECOG PS was 0 in 42 patients $(82.4 \%)$ and 1 in 9 patients $(17.6 \%)$. The most common primary tumor site was the colon in 31 patients $(60.8 \%)$. The most common histology of the primary tumor was moderately differentiated adenocarcinoma in 33 patients $(64.7 \%)$. The most common site of metastasis was multiple sites in 29 patients (56.9\%), followed by metastasis limited to the liver in 13 patients $(25.5 \%)$, and limited to another single region in 9 patients (17.6\%). UGT1A1 status was wild type in 31 patients $(59.6 \%), * 6$ heterozygote in 13 patients $(25 \%), * 28$ heterozygote in six patients $(11.5 \%)$, compound heterozygote in one patient (1.9\%), and unmeasured in one patient (1.9\%). 16 (31.4\%) had received the previous therapy with cetuximab or panitumumab, and 31 patients $(60.8 \%)$ had received the previous therapy with bevacizumab. 


\section{Efficacy}

Tumor response was assessed in 50 patients. Patients were followed for a median of 13.5 months (range 2.4-56.1 months). Median number of treatment cycles was 8.0 (range 1-42 treatment cycles). Median cumulative doses of each agent were: $1780 \mathrm{mg} / \mathrm{m}^{2} /$ day (range $621-2151 \mathrm{mg}$ / $\mathrm{m}^{2} /$ day) for capecitabine; $138.5 \mathrm{mg} / \mathrm{m}^{2} /$ course (range $83.4-151.2 \mathrm{mg} / \mathrm{m}^{2} /$ course) for irinotecan; and $10.0 \mathrm{mg} / \mathrm{kg} /$ course (range $5.2-10.6 \mathrm{mg} / \mathrm{kg} /$ course) for bevacizumab. Median relative dose intensities of each agent were 0.88 (range 0.31-1.08) for capecitabine; 0.92 (range 0.56-1.01) for irinotecan; and 1.00 (range 0.52-1.06) for bevacizumab. Treatment efficacies are summarized in Table 2. Overall, one complete and six partial responses were observed, and response rate was $14.6 \%$ (95\% CI 6.5-28.4\%). Because 25 patients had stable disease, the DCR was $66.7 \%$ (95\% CI 51.5-79.2\%). Median PFS was 5.5 months (95\% CI 4.23-7.40 months) (Fig. 1). Median OS was 13.5 months (95\% CI 11.57-20.23 months) (Fig. 2). Median TTF was 4.5 months (95\% CI 3.97-6.93 months) (Fig. 3). Maximum target lesion response compared to baseline is shown in Fig. 4. Almost all patients (six of seven patients) who showed an increased volume of main lesions over $20 \%$ had used bevacizumab in the first-line therapy. For the sub-analysis of PFS according to first-line treatment, median PFSs were 5.5 months (95\% CI 4.43-10.33) for the bevacizumab group and 6.8 months (95\% CI 3.97-37.33) for the antiEGFR (cetuximab or panitumumab) group (Fig. 5).

\section{Safety}

With the exception of one patient who suffered from grade 4 intestinal pneumonia, all adverse events (AEs) were within grade 3 in this population. Hypertension was the most common Grade 3 adverse event (27.5\%), followed by neutropenia (17.6\%) (Table 3). Anorexia, nausea, vomiting, diarrhea,

Table 2 Efficacy of treatment with XELIRI + Bevacizumab

\begin{tabular}{lc}
\hline Tumor response & Number $(\%)$ \\
\hline CR & $1(2 \%)$ \\
PR & $6(12 \%)$ \\
SD & $25(50 \%)$ \\
PD & $16(32 \%)$ \\
NE & $2(4 \%)$ \\
RR [95\% CI] & $7(14.6 \%)[6.5-28.4]$ \\
DCR $[95 \% \mathrm{CI}]$ & $32(66.7 \%)[51.5-79.2]$ \\
\hline
\end{tabular}

All patients $(N=50)$

$X E L I R I$ xeloda and irinotecan, $C R$ complete response, $P R$ partial response, $S D$ stable disease, $P D$ progressive disease, $N E$ not evaluated, $R R$ response rate, $D C R$ disease control rate, $C I$ confidence interval

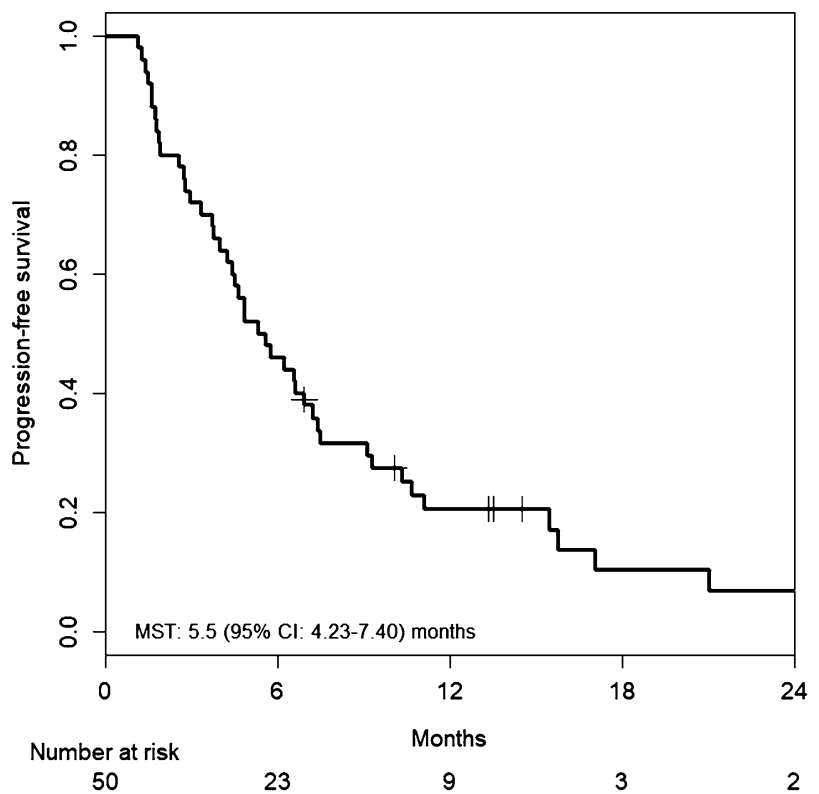

Fig. 1 Kaplan-Meier estimates of progression-free survival (PFS) in the full analysis set of metastatic colorectal cancer (mCRC) patients treated with biweekly CAPIRI + bevacizumab as second-line treatment. Median PFS was 5.5 months (95\% CI 4.23-7.40 months)

and hand-foot syndrome were common at low grades, but at grade 3 showed frequencies of $11.8 \%, 7.8 \%, 2.0 \%, 3.9 \%$, and $3.9 \%$, respectively. Only two patients suffered from grade

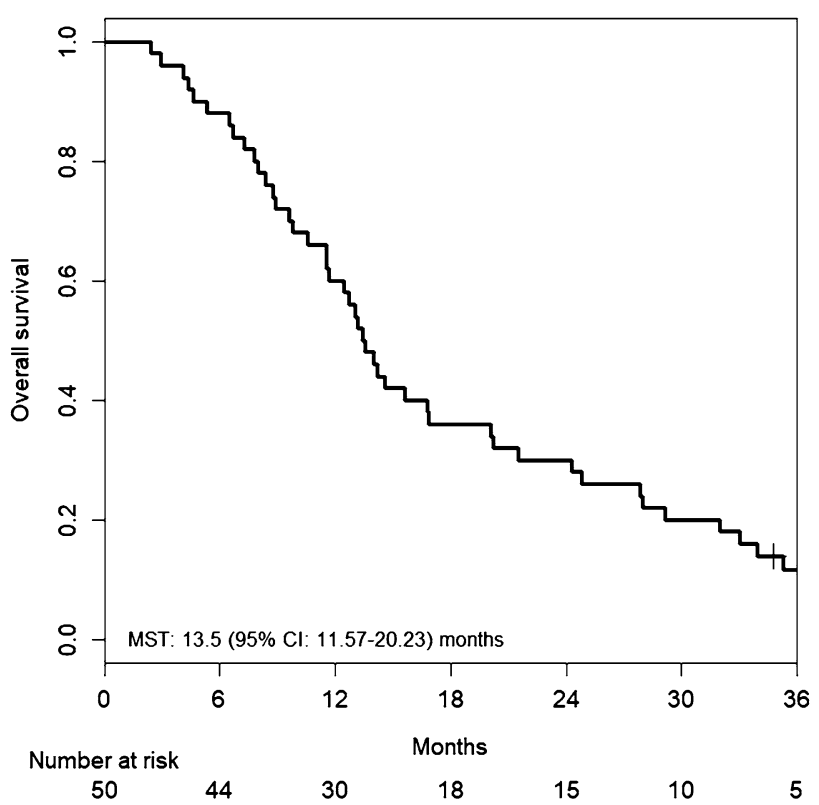

Fig. 2 Kaplan-Meier estimates of overall survival (OS) in the full analysis set of mCRC patients treated with biweekly CAPIRI + bevacizumab as second-line treatment. Median OS was 13.5 months $(95 \%$ CI 11.57-20.23 months) 


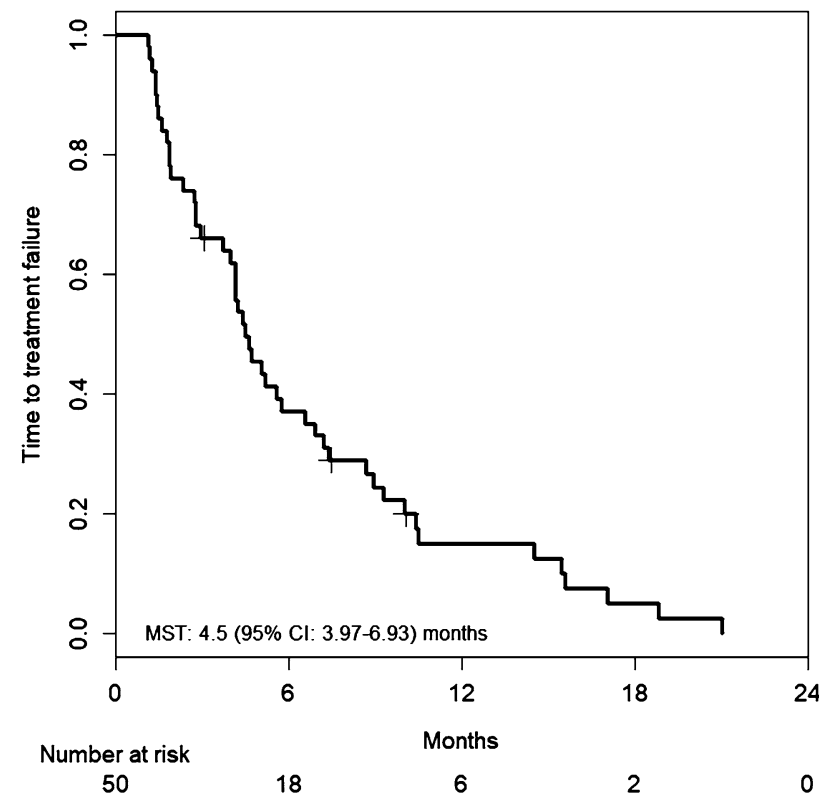

Fig. 3 Kaplan-Meier estimates of time to treatment failure (TTF) in the full analysis set of mCRC patients treated with biweekly CAPIRI+ bevacizumab as second-line treatment. Median TTF was 4.5 months (95\% CI 3.97-6.93 months)

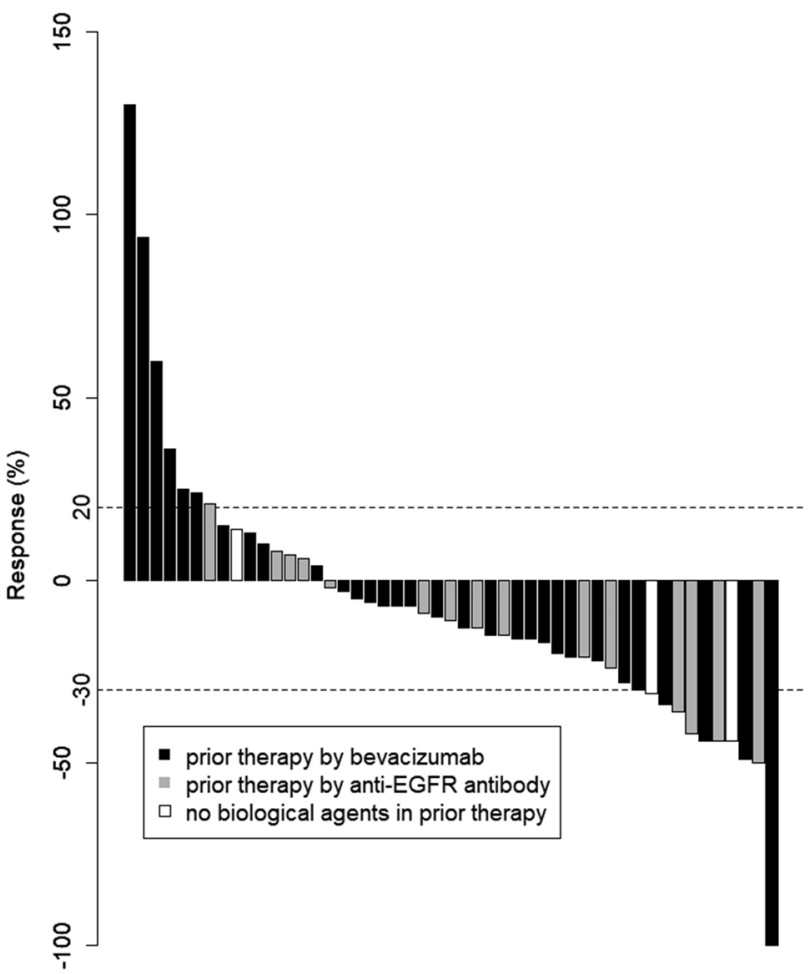

Fig. 4 Waterfall-plot analysis of maximum target lesion response compared to the baseline in patients with mCRC treated with secondline biweekly CAPIRI plus bevacizumab $(N=49)$. Apart from one patient administered anti-EGFR antibody, the other six patients who had target lesions increased over $20 \%$ used bevacizumab as first-line therapy. CAPIRI capecitabine plus irinotecan regimen

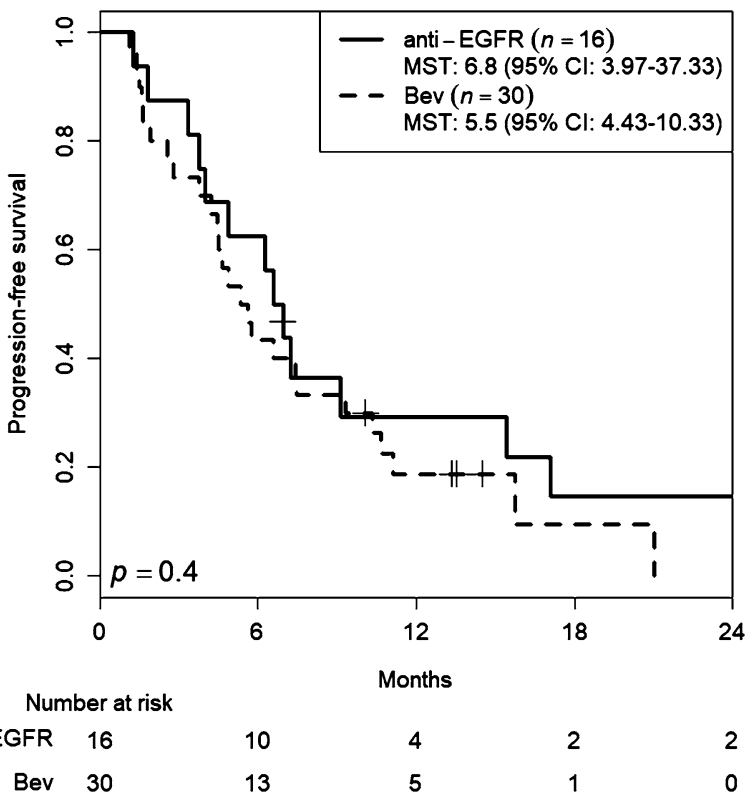

Fig. 5 Kaplan-Meier estimates of progression-free survival (PFS) in the full analysis set of metastatic colorectal cancer (mCRC) patients treated with bevacizumab versus anti-EGFR (cetuximab or panitumumab) as first-line treatment. Median PFS was 5.5 months $(95 \%$ CI 4.43-10.33) and 6.8 months (95\% CI 3.97-37.33) for the bevacizumab group and anti-EGFR group, respectively. Bev bevacizumab. $p=0.4, \log$-rank test

Table 3 Adverse events according to CTCAE version $4.0(N=51)$

\begin{tabular}{lcl}
\hline Adverse events & All grades, $N(\%)$ & grade $3, N(\%)$ \\
\hline Hematological & & \\
Neutropenia & $28(55.0)$ & $9(17.6 \%)$ \\
Anemia & $40(78.4)$ & $1(2.0)$ \\
Thrombocytopenia & $26(51.0)$ & 0 \\
Febrile neutropenia & $2(3.9)$ & $2(3.9)$ \\
Non-hematological & & \\
Anorexia & $32(62.7)$ & $6(11.8)$ \\
Nausea, vomiting & $23(45.1)$ & $4(7.8)$ \\
Diarrhea & $24(47.1)$ & $1(2.0)$ \\
Stomatitis & $12(23.5)$ & $2(3.9)$ \\
Hand-foot syndrome & $23(45.1)$ & $2(3.9)$ \\
Total bilirubin increase & $6(11.8)$ & 0 \\
AST increase & $32(62.7)$ & 0 \\
ALT increase & $20(39.2)$ & 0 \\
Creatinine increase & $11(21.6)$ & 0 \\
Hypertension & $37(72.5)$ & $14(27.5)$ \\
Proteinuria & $24(47.1)$ & $0(0.0)$ \\
Bleeding & $11(21.6)$ & $1(2.0)$ \\
Intestinal pneumonia & $2(3.9)$ & $1(2.0$, grade 4$)$ \\
\hline
\end{tabular}

$A L T$ alanine aminotransferase, $A S T$ aspartate aminotransferase 
3 hand-foot syndrome. No treatment-related mortality was seen among patients in this study.

\section{Discussion}

Irinotecan is one of the key drugs for the treatment of mCRC, along with oxaliplatin [19]. These drugs are often combined with fluorouracil plus leucovorin in regimens such as FOLFIRI or FOLFOX [20, 21]. In recent years, a treatment method replacing intravenous 5-FU with oral fluorinated pyrimidine has been under development, and several methods of administration have been reported using mainly CAPIRI therapy as the first line. Weekly CAPIRI therapy [22], which is mainly administered with CPT-11: $70 \mathrm{mg} / \mathrm{m}^{2}$ every week, and CPT-triweekly CAPIRI therapy administered as $200-300 \mathrm{mg} / \mathrm{m}^{2}$ on day 1 [12]. At the time, we started this trial, no evidence was available regarding CAPIRI plus bevacizumab $(10 \mathrm{mg} / \mathrm{kg})$ as a second-line chemotherapy, especially as a biweekly regimen. Some studies have suggested that irinotecan is associated with significant gastrointestinal toxicities, and several dosages and administration regimens have been investigated to maximize efficacy and tolerability [23]. To the best of our knowledge, this study is one of the first multicenter phase II clinical trials to evaluate the clinical efficacy and safety of biweekly CAPIRI plus $10 \mathrm{mg} /$ $\mathrm{kg}$ bevacizumab as a second-line therapy in patients with mCRC. This study demonstrated that administering capecitabine-irinotecan plus bevacizumab every 2 weeks is feasible and tolerable as a second-line treatment option for patients with mCRC. The target sample size of 50 (43 eligible patients, $10 \%$ ineligible patients) was based on expected and threshold PFSs of 8.0 and 5.1 months, respectively, with $\alpha=0.05$ and $\beta=0.1$. The median PFS of 5.5 months met our primary endpoint, but was slightly shorter than that of reported trials investigating biweekly CAPIRI with bevacizumab treatment as a second-line chemotherapy [16]. However, this was a multicenter study involving general hospitals, with a median age of 66 years, close to the age actually clinical experienced, so our PFS results may be more practically applicable than those from a single high-volume center hospital. Adverse events in the present study were within acceptable rates. Grade 3 febrile neutropenia appeared in two patients (4\%) in our study, similar to rates reported from other studies. We observed grade 3 diarrhea in only one patient $(2 \%)$, much better than the 10-19\% reported by others $[11,16,23]$. Apart from one patient administered anti-EGFR antibody, the other six patients who had increased target lesions by over $20 \%$ had used bevacizumab as a first-line treatment (Fig. 4). For the sub-analysis of PFS, the median PFS was 5.5 months (95\% CI 4.43-10.33) and 6.8 months (95\% CI 3.97-37.33) for bevacizumab group and anti-EGFR (cetuximab or panitumumab) group, respectively, as first-line treatment (Fig. 5).Treatment with biweekly CAPIRI + bevacizumab appears more effective in cases, where bevacizumab is not used in the initial treatment [24].

An Asian phase III trial, the AXEPT study was conducted in the same period as our study [15]. That study compared the efficacy and safety of the mCAPIRI regimen with that of standard FOLFIRI, with or without bevacizumab, in both regimens, as a second-line therapy for mCRC. They concluded that mCAPIRI group is well tolerated and noninferior to FOLFIRI group in terms of OS. Median OS was 16.8 months (95\% CI 15.3-19.1 months) in the mCAPIRI group. In our study, median OS was 13.5 months. The median relative dose intensity in this study was slightly better than that of the AXEPT trial. In the present study, grade $3 / 4$ adverse events of diarrhea, neutropenia, and hand-foot syndrome were less frequent than the standard triweekly CAPIRI regimen $[25,26]$. On the other hand, those were almost the same as in the mCAPIRI group in the AXEPT trial. As only two patients suffered from grade 3 hand-foot syndrome, but it was manageable. In contrast, we selected a bevacizumab dose of $10 \mathrm{mg} / \mathrm{kg}$ on expectation of greater effectiveness in this regimen. A bevacizumab dose of $10 \mathrm{mg} /$ $\mathrm{kg}$ was effective for some cases and tolerable, but the numbers of patients who suffered from hypertension and proteinuria were increased for all grades $(72.5 \%$ and $47.1 \%$, respectively). After we finished enrolling the patients, another phase III trial was reported that compared bevacizumab at $5 \mathrm{mg} / \mathrm{kg}$ and $10 \mathrm{mg} / \mathrm{kg}$ [27]. No significant difference was seen between groups and the frequency of hypertension was increased in the $10 \mathrm{mg} / \mathrm{kg}$ group. Our present study may be feasibly continued and can be administered while maintaining quality of life (QOL) for patients. Patients with unresectable or advanced colorectal cancer sometimes have poor physical condition, such as ECOG PS 1-2. In addition, Japan is now becoming an aging society. We can, therefore, select this regimen comfortably and usefully not only for suitable patients, but for elderly and high-risk patients.

To avoid the grade 3 hypertension, we recommended bevacizumab $5 \mathrm{mg} / \mathrm{kg}$. Based on this limitation, we concluded that biweekly CAPIRI + bevacizumab is well balanced and tolerable in terms of efficacy and safety as a second-line chemotherapy. Biweekly CAPIRI + bevacizumab $(5 \mathrm{mg} / \mathrm{kg})$ could be a replacement for FOLFIRI + bevacizumab in patients with mCRC.

Acknowledgements We would like to thank JSWOGORG for their excellent advice and cooperation, and Mr. Mitsuhiko Ayukawa for his indispensable clinical research coordination.

Funding This study support was NPO; Japan Southwest Oncology Research Support Organization (JSWOGORG). 


\section{Compliance with ethical standards}

Conflict of interest All authors have no conflict of interest to disclose with any companies.

Open Access This article is distributed under the terms of the Creative Commons Attribution 4.0 International License (http://creativeco mmons.org/licenses/by/4.0/), which permits unrestricted use, distribution, and reproduction in any medium, provided you give appropriate credit to the original author(s) and the source, provide a link to the Creative Commons license, and indicate if changes were made.

\section{References}

1. Jemal A, Bray F, Center MM et al (2011) Global cancer statistics. CA Cancer J Clin 61(2):69-90. https://doi.org/10.3322/ caac. 20107

2. Soerjomataram I, Lortet-Tieulent J, Parkin DM et al (2012) Global burden of cancer in 2008: a systematic analysis of disabilityadjusted life-years in 12 world regions. Lancet 380(9856):18401850. https://doi.org/10.1016/S0140-6736(12)60919-2

3. Arnold M, Sierra MS, Laversanne M et al (2017) Global patterns and trends in colorectal cancer incidence and mortality. Gut 66(4):683-691. https://doi.org/10.1136/gutjnl-2015-310912

4. Saltz LB, Clarke S, Diaz-Rubio E et al (2008) Bevacizumab in combination with oxaliplatin-based chemotherapy as first-line therapy in metastatic colorectal cancer: a randomized phase III study. J Clin Oncol 26(12):2013-2019. https://doi.org/10.1200/ JCO.2007.14.9930

5. Cremolini C, Loupakis F, Antoniotti C et al (2015) FOLFOXIRI plus bevacizumab versus FOLFIRI plus bevacizumab as first-line treatment of patients with metastatic colorectal cancer: updated overall survival and molecular subgroup analyses of the openlabel, phase 3 TRIBE study. Lancet Oncol 16(13):1306-1315. https://doi.org/10.1016/S1470-2045(15)00122-9

6. Tabernero J, Van Cutsem E, Lakomy R et al (2014) Aflibercept versus placebo in combination with fluorouracil, leucovorin and irinotecan in the treatment of previously treated metastatic colorectal cancer: prespecified subgroup analyses from the VELOUR trial. Eur J Cancer 50(2):320-331. https://doi.org/10.1016/j. ejca.2013.09.013

7. Tabernero J, Yoshino T, Cohn AL et al (2015) Ramucirumab versus placebo in combination with second-line FOLFIRI in patients with metastatic colorectal carcinoma that progressed during or after first-line therapy with bevacizumab, oxaliplatin, and a fluoropyrimidine (RAISE): a randomised, double-blind, multicentre, phase 3 study. Lancet Oncol 16(5):499-508. https://doi. org/10.1016/S1470-2045(15)70127-0

8. Heinemann V, von Weikersthal LF, Decker T et al (2014) FOLFIRI plus cetuximab versus FOLFIRI plus bevacizumab as first-line treatment for patients with metastatic colorectal cancer (FIRE-3): a randomised, open-label, phase 3 trial. Lancet Oncol 15(10):1065-1075. https://doi.org/10.1016/S1470-2045(14)70330 $-4$

9. Schwartzberg LS, Rivera F, Karthaus M et al (2014) PEAK: a randomized, multicenter phase II study of panitumumab plus modified fluorouracil, leucovorin, and oxaliplatin (mFOLFOX6) or bevacizumab plus mFOLFOX6 in patients with previously untreated, unresectable, wild-type KRAS exon 2 metastatic colorectal cancer. J Clin Oncol 32(21):2240-2247. https://doi. org/10.1200/JCO.2013.53.2473
10. Elez E, Argiles G, Tabernero J (2015) First-line treatment of metastatic colorectal cancer: interpreting FIRE-3, PEAK, and CALGB/ SWOG 80405. Curr Treat Options Oncol 16(11):52. https://doi. org/10.1007/s11864-015-0369-x

11. Ducreux M, Adenis A, Pignon JP et al (2013) Efficacy and safety of bevacizumab-based combination regimens in patients with previously untreated metastatic colorectal cancer: final results from a randomised phase II study of bevacizumab plus 5-fluorouracil, leucovorin plus irinotecan versus bevacizumab plus capecitabine plus irinotecan (FNCLCC ACCORD 13/0503 study). Eur J Cancer 49(6):1236-1245. https://doi. org/10.1016/j.ejca.2012.12.011

12. Fuchs CS, Marshall J, Mitchell E et al (2007) Randomized, controlled trial of irinotecan plus infusional, bolus, or oral fluoropyrimidines in first-line treatment of metastatic colorectal cancer: results from the BICC-C Study. J Clin Oncol 25(30):4779-4786. https://doi.org/10.1200/JCO.2007.11.3357

13. CH Kohne J Greve De JT Hartmann et al. 2008 Irinotecan combined with infusional 5-fluorouracil/folinic acid or capecitabine plus celecoxib or placebo in the first-line treatment of patients with metastatic colorectal cancer. EORTC study 40015 Ann Oncol 195920926 10.1093/annonc/mdm544.

14. Hamamoto Y, Yamaguchi T, Nishina T et al (2014) A phase I/II study of XELIRI plus bevacizumab as second-line chemotherapy for Japanese patients with metastatic colorectal cancer (BIX study). Oncologist 19(11):1131-1132. https://doi.org/10.1634/ theoncologist.2014-0159

15. RH Xu K Muro S Morita et al. 2018 Modified XELIRI (capecitabine plus irinotecan) versus FOLFIRI (leucovorin, fluorouracil, and irinotecan), both either with or without bevacizumab, as second-line therapy for metastatic colorectal cancer (AXEPT): a multicentre, open-label, randomised, non-inferiority, phase 3 trial Lancet Oncol 10.1016/S1470-2045(18)30140-2.

16. Suenaga M, Mizunuma N, Matsusaka S et al (2015) A phase I/ II study of biweekly capecitabine and irinotecan plus bevacizumab as second-line chemotherapy in patients with metastatic colorectal cancer. Drug Des Devel Ther 9:1653-1662. https://doi. org/10.2147/DDDT.S80449

17. Hazama S, Maeda H, Iwamoto S et al (2016) A phase II study of XELOX and cetuximab as first-line therapy in patients with KRAS wild type metastatic colorectal cancer (FLEET2 study). Clin Colorectal Cancer 15(4):329-336. https://doi.org/10.1016/j. clcc.2016.07.003

18. Jordan K, Kellner O, Kegel T et al (2004) Phase II trial of capecitabine/irinotecan and capecitabine/oxaliplatin in advanced gastrointestinal cancers. Clin Colorectal Cancer 4(1):46-50

19. Grothey A, Sargent D (2005) Overall survival of patients with advanced colorectal cancer correlates with availability of fluorouracil, irinotecan, and oxaliplatin regardless of whether doublet or single-agent therapy is used first line. J Clin Oncol 23(36):94419442. https://doi.org/10.1200/JCO.2005.04.4792

20. Tournigand C, Andre T, Achille E et al (2004) FOLFIRI followed by FOLFOX6 or the reverse sequence in advanced colorectal cancer: a randomized GERCOR study. J Clin Oncol 22(2):229-237. https://doi.org/10.1200/JCO.2004.05.113

21. Grothey A, Sargent D, Goldberg RM et al (2004) Survival of patients with advanced colorectal cancer improves with the availability of fluorouracil-leucovorin, irinotecan, and oxaliplatin in the course of treatment. J Clin Oncol 22(7):1209-1214. https://doi. org/10.1200/JCO.2004.11.037

22. Borner MM, Bernhard J, Dietrich D et al (2005) A randomized phase II trial of capecitabine and two different schedules of irinotecan in first-line treatment of metastatic colorectal cancer: efficacy, quality-of-life and toxicity. Ann Oncol 16(2):282-288. https://doi.org/10.1093/annonc/mdi047 
23. Pectasides D, Papaxoinis G, Kalogeras KT et al (2012) XELIRI-bevacizumab versus FOLFIRI-bevacizumab as firstline treatment in patients with metastatic colorectal cancer: a Hellenic Cooperative Oncology Group phase III trial with collateral biomarker analysis. BMC Cancer 12:271. https://doi. org/10.1186/1471-2407-12-271

24. Bennouna J, Sastre J, Arnold D et al (2013) Continuation of bevacizumab after first progression in metastatic colorectal cancer (ML18147): a randomised phase 3 trial. Lancet Oncol 14(1):2937. https://doi.org/10.1016/S1470-2045(12)70477-1

25. Ota M, Tatsumi K, Suwa $\mathrm{H}$ et al (2014) The effect of pyridoxine for prevention of hand-foot syndrome in colorectal cancer patients with adjuvant chemotherapy using capecitabine: a randomized study. Hepatogastroenterology 61(132):1008-1013

26. Patt YZ, Lee FC, Liebmann JE et al (2007) Capecitabine plus 3-weekly irinotecan (XELIRI regimen) as first-line chemotherapy for metastatic colorectal cancer: phase II trial results. Am J Clin Oncol 30(4):350-357. https://doi.org/10.1097/COC.0b013e3180 $4 \mathrm{~b} 40 \mathrm{bb}$

27. Iwamoto S, Takahashi T, Tamagawa $\mathrm{H}$ et al (2015) FOLFIRI plus bevacizumab as second-line therapy in patients with metastatic colorectal cancer after first-line bevacizumab plus oxaliplatinbased therapy: the randomized phase III EAGLE study. Ann Oncol 26(7):1427-1433. https://doi.org/10.1093/annonc/mdv197

Publisher's Note Springer Nature remains neutral with regard to jurisdictional claims in published maps and institutional affiliations.

\section{Affiliations}

\section{Nobuaki Suzuki ${ }^{1}$ (D) Shoichi Hazama ${ }^{1,2} \cdot$ Takeshi Nagasaka $^{3} \cdot$ Hiroaki Tanioka $^{3}$ - Yasuo Iwamoto ${ }^{4} \cdot$ Yuji Negoro $^{5}$. Masami Yamauchi ${ }^{6} \cdot$ Michiya Kobayashi $^{7} \cdot$ Hiroshi Okuda $^{8} \cdot$ Noriaki Fujishima $^{9} \cdot$ Taku Nishimura $^{10}$. Naoki Yamanaka ${ }^{11} \cdot$ Kazuhiro Toyota $^{12} \cdot$ Yoshiko Mori $^{13} \cdot$ Yuki Nakagami $^{1,2} \cdot$ Mototsugu Shimokawa $^{14}$. Hiroaki Nagano ${ }^{1} \cdot$ Masazumi Okajima $^{15}$}

1 Department of Gastroenterological, Breast and Endocrine Surgery, Yamaguchi University Graduate School of Medicine, 1-1-1 Minami-Kogushi, Ube 755-8505, Japan

2

Department of Translational Research and Developmental Therapeutics Against Cancer, Yamaguchi University School of Medicine, Ube, Japan

3 Department of Clinical Oncology, Kawasaki Medical School, Kurashiki, Japan

4 Department of Medical Oncology, Hiroshima City Hiroshima Citizens Hospital, Hiroshima, Japan

5 Department of Medical Oncology, Kochi Health Sciences Center, Kochi, Japan

6 Division of Clinical Oncology, Hiroshima Prefectural Hospital, Hiroshima, Japan

7 Cancer Treatment Center, Kochi Medical School Hospital, Kochi, Japan

8 Surgery and Endoscopic Surgery, Onomichi General Hospital, Onomichi, Japan
9 Department of Surgery, Fukuda Clinic of Internal Medicine, Heart and Digestive, Kochi, Japan

10 Department of Surgery, Kokura Memorial Hospital, Kokura, Japan

11 Department of Surgery, Japanese Red Cross Yamaguchi Hospital, Yamaguchi, Japan

12 Department of Surgery, National Hospital Organization Higashihiroshima Medical Center, Hiroshima, Japan

13 Department of Gastroenterological Surgery, Okayama University Graduate School of Medicine, Dentistry and Pharmaceutical Sciences, Okayama, Japan

14 Cancer Biostatistics Laboratory, Clinical Research Institute, National Kyushu Cancer Center, Fukuoka, Japan

15 Department of Surgery, Hiroshima City Hiroshima Citizens Hospital, Hiroshima, Japan 